\title{
AV reentrant and idiopathic ventricular double tachycardias: complicated interactions between two tachycardias
}

\author{
T Washizuka, S Niwano, K Tsuchida, Y Aizawa
}

First Department of Internal Medicine, Niigata University School of Medicine, Asahimachi 1-754, Niigata 951, Japan $\mathrm{T}$ Washizuka S Niwano

K Tsuchida

Y Aizawa

Correspondence to: Dr Washizuka. email: washi@med. niigata-u.ac.jp 2 September 1998
Accepted for publication

\section{Abstract}

An electrophysiological study was performed in a 61 year old man with WolffParkinson-White (WPW) syndrome. At baseline, neither ventricular nor supraventricular tachycardias could be induced. During isoprenaline infusion, ventricular tachycardia originating from the right ventricular outflow tract (RVOT) with a cycle length of $280 \mathrm{~ms}$ was induced and subsequently atrioventricular reentrant tachycardia (AVRT) with a cycle length of $300 \mathrm{~ms}$ using an accessory pathway in the left free wall appeared. During these tachycardias, AVRT was entrained by ventricular tachycardia. The earliest ventricular activation site during the ventricular tachycardia was determined to be the RVOT site and a radiofrequency current at $30 \mathrm{~W}$ successfully ablated the ventricular tachycardia at this site. The left free wall accessory pathway was also successfully ablated during right ven-

A RBBB tachycardia

B Pre-ablation

C Postablation
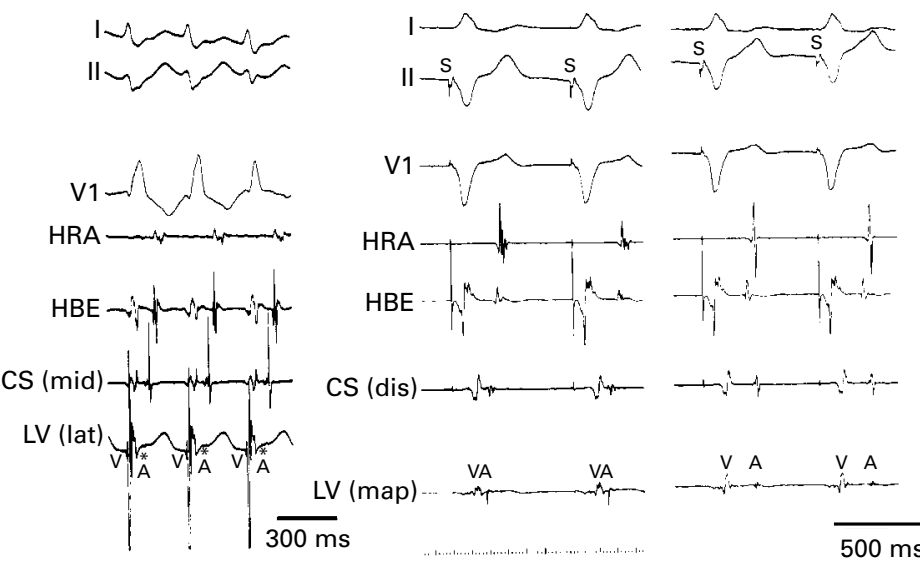

Figure 1 Intracardiac recordings during RBBB tachycardia and during ventricular pacing before and after ablation. Surface leads I, II, and V1 are shown with intracardiac electrograms from the high right atrium (HRA), the His bundle electrogram recording site ( $H B E)$, coronary sinus (CS), and the left ventricle ( $L V)$. (A) AVRT with RBBB morphology at a cycle length of $300 \mathrm{~ms}$ are seen. The earliest site of retrograde atrial conduction was observed at the left lateral site of the subvalvar portion of the mitral valve. The asterisks show the retrograde atrial activity. $(B)$ and $(C)$ The sequence of the ventriculoatrial conduction changed after the ablation. mid, middle portion; lat, lateral portion; dis, distal portion; map, mapping catheter. tricular pacing. The coexistence of WPW syndrome and cathecolamine sensitive ventricular tachycardia originating from the RVOT has rarely been reported. Furthermore, the tachycardias were triggered by previous tachycardias.

(Heart 1999;81:318-320)

Keywords: double tachycardia; Wolff-Parkinson-White syndrome; radiofrequency catheter ablation; arrhythmias

Double tachycardia is classically defined as the simultaneous occurrence of atrial and ventricular tachycardias, ${ }^{12}$ or junctional and ventricular tachycardias. This type of tachycardia has been described in digitalis intoxication, generally in patients with left ventricular dysfunction. ${ }^{12}$ Exercise or cathecolamine induced double tachycardia has also been reported ${ }^{3}$ but the coexistence of atrioventricular reentrant tachycardia (AVRT) and ventricular tachycardia originating in the right ventricular outflow tract (RVOT) has not been reported. We describe a patient with WolffParkinson-White syndrome who demonstrated both AVRT and ventricular tachycardia. Moreover, these tachycardias appeared simultaneously and were triggered by previous tachycardias.

\section{Case report}

A 61 year old man with WPW syndrome had episodes of palpitation and dizziness. During an episode of palpitation, the ECG documented two types of wide QRS tachycardias with a heart rate of 190-200 beats/min. Despite prophylactic oral verapamil (120 mg/day) symptomatic episodes continued. $\mathrm{He}$ was referred to our hospital for electrophysiological evaluation. Surface ECG showed sinus rhythm with a short PQ interval $(0.10$ seconds $)$ although the delta wave was not clear. No underlying heart disease was found by standard examination.

After obtaining informed consent, electrophysiological study was performed in the postabsorptive, non-sedated state. There was no detectable abnormality in sinus nodal function. The atrial extrastimuli and rapid pacing from the high right atrium revealed a positive 


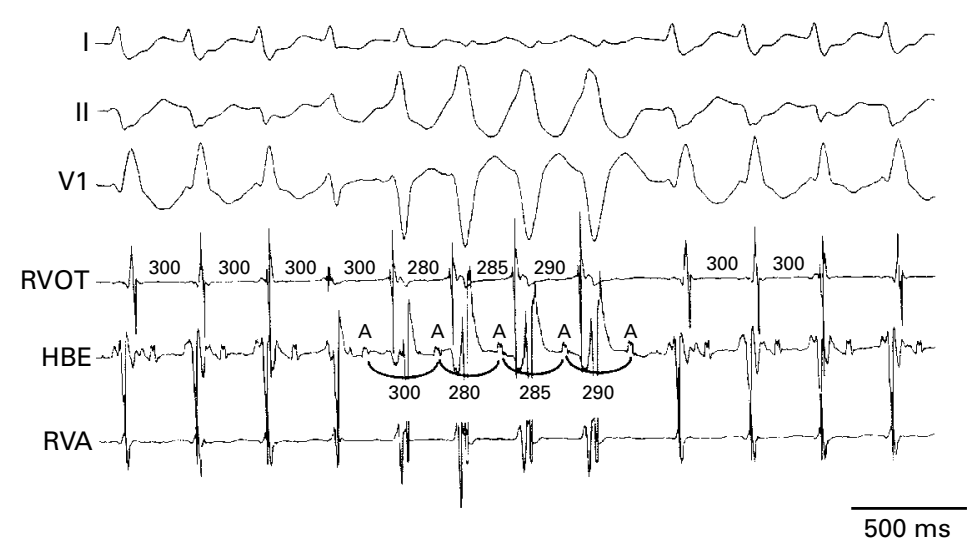

Figure 2 Intracardiac recording during double tachycardia. Surface leads I, II, and VI are shown with intracardiac electrograms from the RVOT, the His bundle electrogram recording site ( $H B E$ ), and the right ventricular apex (RVA). AVRT with RBBB morphology at a cycle length of $300 \mathrm{~ms}$ and non-sustained ventricular tachycardia with LBBB morphology at a cycle length of 280-290 ms are seen. Transient entrainment phenomenon of the AVRT by non-sustained ventricular tachycardia is observed.

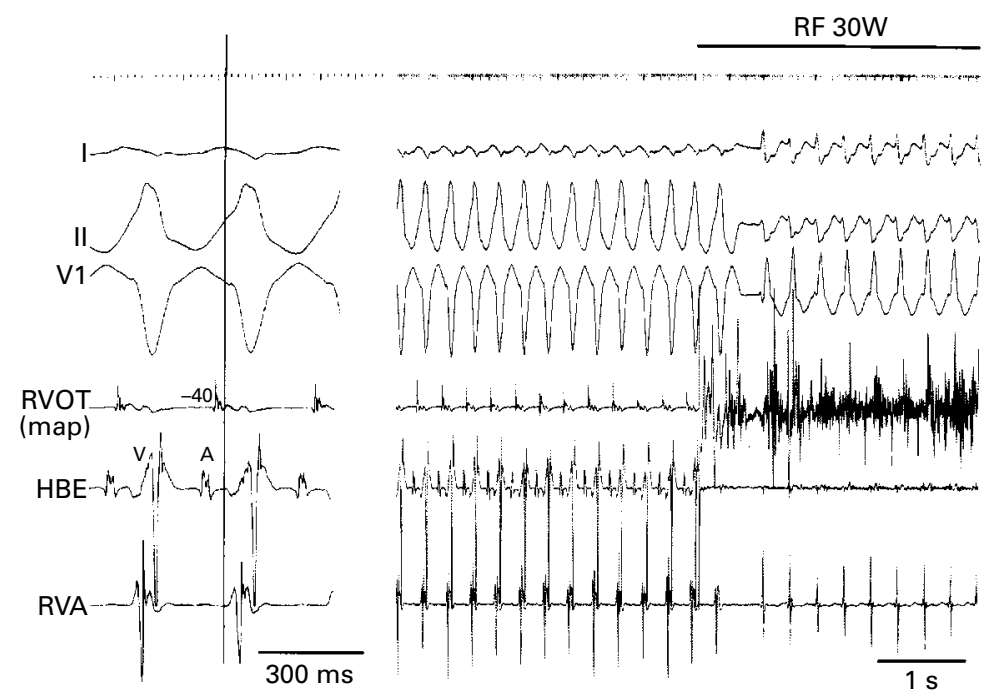

Figure 3 Radiofrequency catheter ablation $(R F)$ of the origin of ventricular tachycardia in the right ventricular outflow tract. (Left) The earliest activation site was determined. The local electrogram at the site preceded the onset of the QRS complex by $40 \mathrm{~ms}$. (Right) $R F$ current at $30 \mathrm{~W}$ was delivered to the earliest activation site, the ventricular tachycardia was successfully ablated and the AVRT appeared. map, mapping catheter; HBE, His bundle electrogram; RVA, right ventricular apex.

delta wave in lead V1, and the atrioventricular (AV) conduction time was constant. The ventriculoatrial (VA) conduction time was constant over a wide range of extrastimulus coupling intervals from the right ventricular apex. The earliest ventricular activation site during sinus rhythm and the earliest atrial activation site during ventricular pacing were observed at the distal recording site of the coronary sinus catheter, which was positioned at the left lateral free wall (fig 1B). At baseline, neither supraventricular nor ventricular tachycardia could be induced by programmed stimulation. During isoprenaline infusion $(0.33 \mu \mathrm{g} / \mathrm{min})$ the wide QRS tachycardia with a configuration of left bundle branch block (LBBB) at a cycle length of 280-290 ms occurred spontaneously. This tachycardia lasted 1-3 seconds and repeated incessantly.
Subsequently, the wide QRS tachycardia with a configuration of right bundle branch block (RBBB) at a cycle length of $300 \mathrm{~ms}$ appeared.

The earliest site of ventricular activation was observed at the outflow tract of the right ventricle and the earliest site of retrograde conduction was the distal recording site of the coronary sinus catheter in the LBBB-type tachycardia. Moreover, AV dissociation was observed during this tachycardia. During the RBBB tachycardia, the AV node-His bundle system was utilised for antegrade conduction and the earliest site of retrograde conduction was observed at the left lateral site of the mitral valve (fig 1A). The RBBB tachycardia was entrained by the LBBB tachycardia (fig 2). The LBBB tachycardia was considered to be a cathecolamine sensitive ventricular tachycardia that originated from the right ventricular outflow tract, while the RBBB tachycardia was orthodromic AVRT using the left lateral accessory pathway.

By increasing the dosage of isoprenaline $(0.5 \mu \mathrm{g} / \mathrm{min})$ the duration of ventricular tachycardia was prolonged and finally sustained. Radiofrequency current at $30 \mathrm{~W}$ was applied to the earliest ventricular activation site in the right ventricular outflow tract through a $7 \mathrm{~F}$ quadripolar electrode catheter with a $4 \mathrm{~mm}$ tip electrode (Cordis/Webster, Boston Scientific International, Boston, Massachusetts, USA). The ventricular tachycardia was terminated within seconds and the AVRT appeared (fig 3). We then performed detailed endocardial mapping along the mitral valve. The earliest ventricular activation site during sinus rhythm and the earliest atrial retrograde activation site during right ventricular pacing were determined in the left lateral site of the mitral valve, and the accessory pathway was successfully ablated at this site (fig 1). After these ablation procedures, neither ventricular tachycardia nor AVRT was induced by isoprenaline or programmed stimulation.

\section{Discussion}

Double tachycardia is a relatively uncommon type of tachycardia ${ }^{1-4}$ and the coexistence of cathecolamine sensitive ventricular tachycardia and AVRT have rarely been reported. In our case, although neither ventricular nor supraventricular tachycardia was induced by programmed stimulation at basal state, isoprenaline infusion provoked ventricular tachycardia and subsequently AVRT. Enhanced automaticity was considered to be the mechanism of ventricular tachycardia, because the site of ventricular tachycardia origin was localised at the RVOT and the ventricular tachycardia appeared spontaneously during isoprenaline infusion. Moreover, isoprenaline infusion is considered to enable the AVRT to be sustained through the enhancement of AV conduction. While sustained AVRT increases the adrenergic tone, it may result in induction of the ventricular tachycardia originating from the RVOT. Thus each tachycardia may be triggered by a previous one, especially in clinical situations. 
During endocardial mapping, we had difficulty with the practical procedure owing to a complicated mixture of AVRT and nonsustained cathecolamine sensitive ventricular tachycardia. By increasing the isoprenaline dosage, the ventricular tachycardia became sustained without being haemodynamically unstable. Therefore, we could perform accurate endocardial mapping. In ventricular double tachycardias ${ }^{5}$ endocardial mapping may be more difficult than in our case. Precise endocardial mapping is essential for successful ablation procedures, exclusion of one tachycar- dia from the complicated coexistence of double tachycardia is important.

1 Castellanos A Jr, Azan L, Calvino JM. Simultaneous tachycardias. Am Heart 7 1960;59:358-73.

2 Chowdhry IH, Hariman RJ, Gomes JA, et al. Transient digitoxic double tachycardia. Chest 1983;83:686-7.

3 Benson DW Jr, Gallagher JJ, Sterba R, et al. Cathecolamine induced double tachycardia: case report in a child. $P A C E$ 1980;3: 96-103.

4 Eldar M, Belhassen B, Hod H, et al. Exercise-induced double (atrial and ventricular) tachycardia: a report of three cases. 7 Am Coll Cardiol 1989;14:1376-81.

5 Niwano S, Aizawa Y, Tamura M, et al. Intraventricular concealed double tachycardia: a case report. Intern Med 1992; 31:227-231.

\section{IMAGES IN CARDIOLOGY}

\section{Inoue balloon dilatation of a mitral valve bioprosthesis}
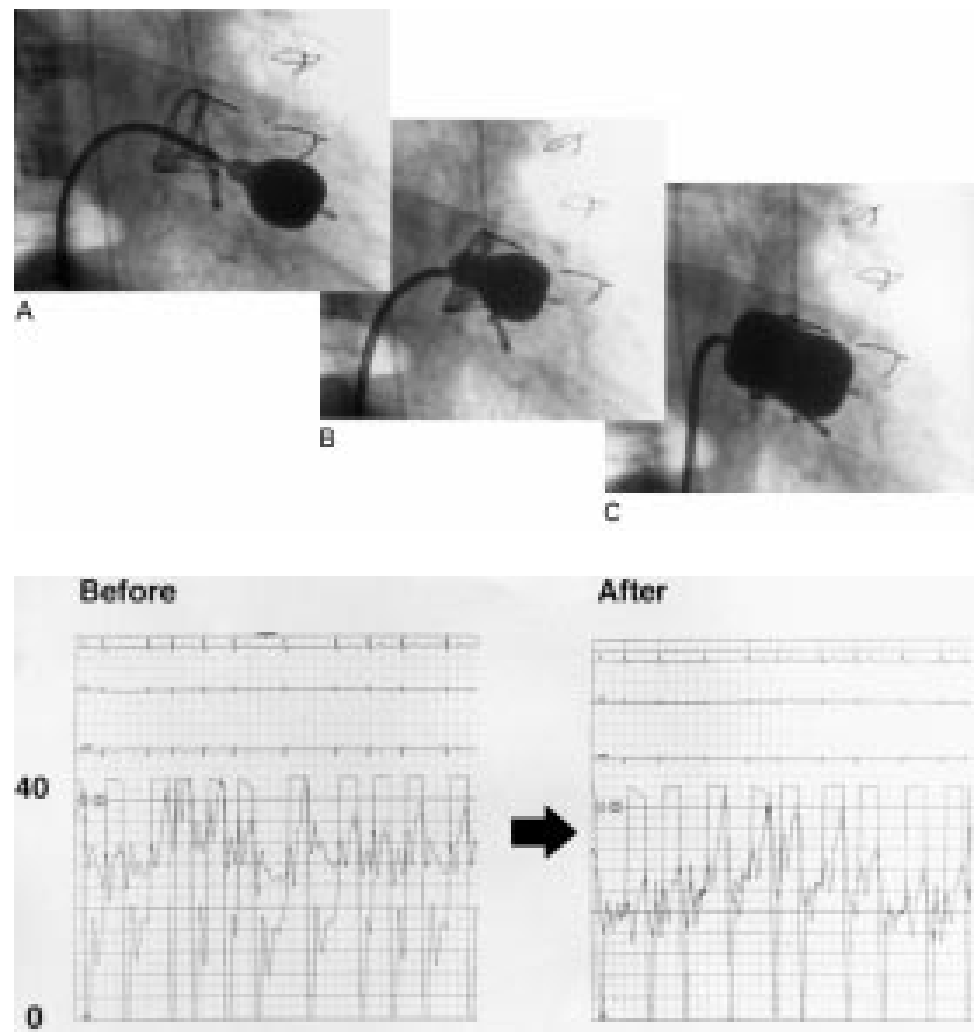

A 54 year old female patient had severely calcific rheumatic mitral stenosis causing limiting exertional dyspnoea in 1988; she was treated by implantation of an Edwards pericardial valve with resolution of symptoms. In 1997 she developed recurrent breathlessness owing to stenosis of the bioprosthetic valve with an estimated valve area of $0.9 \mathrm{~cm}^{2}$. She was treated with Inoue balloon dilatation of the bioprosthetic valve. Top figure (A) shows the balloon positioned through the mitral valve in the left ventricle. The balloon is partially inflated (the distal portion opening first). The balloon is then withdrawn to be positioned across the mitral bioprosthetic valve (B), and then fully inflated (C). Following this procedure the gradient between left atrial and left ventricular pressure is almost completely abolished (bottom). Left venticulography confirmed that balloon dilatation had not caused mitral regurgitation.

The literature regarding balloon dilatation of stenosed bioprothetic valves is limited to case reports and small series. Although the medium and long term outcome is less clear than for native mitral stenosis, balloon dilatation of bioprostheses may be a way to delay or avoid the hazards of repeat mitral surgery in some patients.

PETER F LUDMAN MICHAEL P I PITT 